RICYDE. Revista Internacional de Ciencias del Deporte doi: $10.5232 /$ ricyde

Rev. int. cienc. deporte

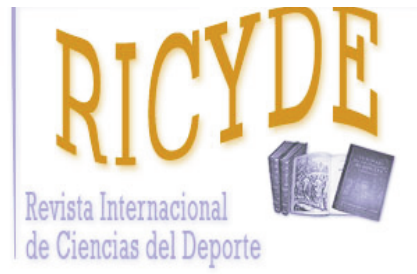

RICYDE. Revista Internacional de Ciencias del Deporte VOLUMEN XIII - AÑO XIII

Páginas:162-171 ISSN:1885-3137

Número 48 - Abril - 2017

\title{
Riesgo de adicción al ejercicio en triatletas hombres amateur y su relación con variables de entrenamiento \\ Risk of exercise addiction among male amateur triathletes and its relationship with training variables
}

\author{
Pedro L. Valenzuela, Fernando Arriba-Palomero \\ Universidad de Alcalá. España
}

\begin{abstract}
Resumen
Debido a la creciente popularidad del triatlón a nivel amateur el objetivo de este estudio fue evaluar el potencial adictivo de este deporte, así como analizar si determinadas variables de entrenamiento podrían ser consideradas factores de riesgo. Atendiendo a la escala de dependencia al ejercicio revisada (Exercise Dependence Scale-Revised, EDS-R), 93 triatletas varones amateur (34.5 \pm 9.4 años) fueron divididos en no dependientes asintomáticos (NDA, 31.2\%), no dependientes sintomáticos (NDS, 60.2\%) o en riesgo de dependencia (ERD, 8.6\%). Se halló una correlación moderada entre el volumen de entrenamiento y la puntuación en la escala EDS-R, entrenando los sujetos ERD más horas y sesiones a la semana que los sujetos NDA. El porcentaje de sujetos clasificados como ERD fue mayor según aumentaba la distancia de competición $(0.0,7.1$ y $30.0 \%$ de los triatletas de corta, media y larga distancia, respectivamente), siendo la puntuación en la EDS-R de los triatletas de larga distancia significativamente mayor que la de los de media y corta distancia. Estos resultados muestran el potencial adictivo del triatlón, en concreto en larga distancia, y resaltan la importancia del adecuado control psicológico en esta población.
\end{abstract}

Palabras clave: Adicción al ejercicio; triatlón; volumen de entrenamiento.

\begin{abstract}
Due to the growing popularity of triathlon at an amateur level the aim of this study was to evaluate the addictive potential of this sport as well as to analyze if particular training variables could be considered as risk factors. Attending to the Exercise Dependence Scale-Revised (EDS-R), 93 male amateur triathletes (34.5 \pm 9.4 years old) were classified as Non-Dependent Asymptomatic (NDA, 31.2\%), Non-Dependent Symptomatic (NDS, 60.2\%) or At-Risk of Dependence (ARD, 8.6\%). A moderate correlation between training volume and the EDS-R score was found, training ARD more hours and sessions per week than NDA. A greater percentage of subjects classified as ARD was found as race distance increased $(0.0,7.1$ and 30.0 $\%$ for short, half and long distance, respectively), being the EDS-R score of long distance triathletes higher than those of half and short distance. These results show the addictive potential of triathlon, specifically in long distance, and highlight the importance of an optimal psychological assessment in this population.
\end{abstract}

Key words: Exercise addiction; triathlon; training volume. 
Valenzuela, P. L. y Arriba-Palomero, F. (2017). Riesgo de adicción al ejercicio en triatletas hombres amateur y su relación con variables de entrenamiento. RICYDE. Revista internacional de ciencias del deporte, 48(13), 162-171. https://doi.org/10.5232/ricyde2017.04806

\section{Introducción}

T a popularidad del triatlón se ha disparado en los últimos años, siendo alcanzadas las 140000 licencias federativas en EEUU (Youngman y Simpson, 2014) y sufriendo esta cifra un aumento de un 380\% en los últimos diez años en España (29739 licencias en 2015, dato obtenido a través del Consejo Superior de Deportes de España). La práctica de este deporte requiere un alto esfuerzo tanto a nivel físico (Laursen, 2011) como psicológico (Heazlewood y Burke, 2011) por parte de los triatletas, aunque no supone en general riesgos para los triatletas bien entrenados (Vleck, Millet, y Alves, 2014).

Los beneficios psicológicos (Penedo y Dahn, 2005) y fisiológicos (Fiuza-Luces, Garatachea, Berger, y Lucia, 2013) del deporte son ampliamente conocidos. Sin embargo, cuando éste es realizado de forma compulsiva y excesiva puede conllevar la aparición de adicción al ejercicio, con importantes consecuencias tanto físicas como psicológicas y sociales. La adicción al ejercicio, pese a no estar incluida en el último manual diagnóstico y estadístico de los trastornos mentales (Diagnostic and Statistical Manual of Mental Disorders, DSM) debido a falta de evidencia (Szabo, 2005), puede ser considerada una adicción conductual, ya que al igual que ocurre con otras adicciones conductuales como la adicción a las compras o a la televisión, la persona adicta al ejercicio pierde el control sobre dicho comportamiento o conducta por el placer que le genera y lo realiza de forma compulsiva a pesar de las posibles consecuencias negativas (Berczik, Szabó, Griffiths, Kurimay, Kun, Urbán, y Demetrovics, 2012).

Cuando el ejercicio se convierte en una adicción los sujetos sufren a menudo daños corporales por los niveles descontrolados de ejercicio, así como daños a nivel psicológico y físiológico incluyendo ansiedad, irritabilidad, fasciculaciones musculares, incapacidad para conciliar el sueño o para concentrarse y otros síntomas asociados al síndrome de abstinencia (Griffiths, 1997; Berczik, Szabó, Griffiths, Kurimay, Kun, Urbán, y Demetrovics, 2012; Szabo, Griffiths, de La Vega Marcos, Mervó, y Demetrovics, 2015). Por lo tanto, la calidad de vida de estas personas se ve altamente afectada.

La prevalencia de adicción al ejercicio varía según la población analizada. Así, mientras se ha determinado una prevalencia del $2-3 \%$ en la población general o moderadamente activa (Sussman, Lisha, y Griffiths, 2011; Monok, Berczik, Urbán, Szabo, Griffiths, Farkas, Magi, Eisinger, Kurimay, Kökönyei, Kun, Paksi y Demetrovics, 2012), este valor aumenta hasta un $42 \%$ en jóvenes que entrenaban en un gimnasio (Lejoyeux, Avril, Richoux, Embouazza, y Nivoli, 2008) o hasta el 34\% en deportistas de élite de diferentes disciplinas (McNamara y McCabe, 2012).

En el caso de los deportes de resistencia también se han encontrado resultados variables, desde el 3.4\% encontrado en ultra-maratonianos (Allegre, Therme, y Griffiths, 2007) hasta el $26.2 \%$ encontrado en hombres corredores de nivel variable (Slay, Hayaki, Napolitano, y Brownell, 1998). De igual forma, en triatlón los estudios muestran resultados dispares aunque en todo caso preocupantes. Así, mientras Magee y col (2016) encontraron una prevalencia de riesgo de adicción del $8.4 \%$, otros autores han mostrado una prevalencia superior al $20 \%$ (Blaydon, Lindner, y Kerr, 2002; Ruby, 2008). Esta discrepancia podría deberse a diferencias en las características de la muestra como el sexo, la distancia en la que competían los triatletas (corta, media o larga distancia) o a la inclusión de forma conjunta de deportistas amateur y profesionales. 
Valenzuela, P. L. y Arriba-Palomero, F. (2017). Riesgo de adicción al ejercicio en triatletas hombres amateur y su relación con variables de entrenamiento. RICYDE. Revista internacional de ciencias del deporte, 48(13), 162-171. https://doi.org/10.5232/ricyde2017.04806

Al presentar cada distancia de competición en triatlón unas características y unos requerimientos físicos y psicológicos específicos (Laursen, 2011), no es conveniente estudiar el riesgo de adicción en triatletas de forma independiente a esta variable. Hasta donde sabemos, sólo un estudio (Youngman y Simpson, 2014) ha analizado el riesgo de adicción en triatletas atendiendo a la distancia de competición de los mismos, encontrando mayor prevalencia en aquellos que competían en media y larga distancia que en los de corta.

Debido a la creciente popularidad del triatlón a nivel amateur en los últimos años en España, el objetivo de este estudio fue analizar la prevalencia de riesgo de adicción al ejercicio en esta población. Además, se trató de determinar si la distancia en la que los triatletas compiten u otras variables como la experiencia o el volumen de entrenamiento podrían estar relacionadas con un mayor riesgo de adicción.

\section{Métodos}

\section{Participantes}

Con el objetivo de analizar una muestra lo más homogénea posible los criterios de inclusión para participar en el estudio fueron ser hombre, mayor de edad, deportista no profesional, tener una experiencia mínima en el triatlón de tres años, haber competido en al menos un triatlón en el último año y entrenar un mínimo de una sesión por semana de cada una de las disciplinas que componen este deporte (natación, ciclismo y carrera).

Los sujetos fueron reclutados de tres clubes de triatlón diferentes de la zona centro de España, presentando un nivel socio-económico similar. 93 hombres (34,5 $\pm 9,4$ años) cumplieron los requisitos establecidos y fueron por tanto incluidos en el presente estudio tras firmar el correspondiente consentimiento informado. El presente estudio ha sido aceptado por el Comité de Ética de la Universidad de Alcalá.

\section{Instrumentos}

Se utilizó un cuestionario anónimo que incluía la escala de dependencia al ejercicio traducida al español (Exercise Dependence Scale, EDS-R; Sicilia y González-Cutre, 2011), la cual ha sido desarrollada atendiendo al DSM.

La EDS-R contiene 21 ítems referidos a 7 comportamientos o factores relacionados con el ejercicio y cuyas respuestas van de 1 (nunca) a 6 (siempre). En concreto, los ítems se refieren a la tolerancia (necesidad de aumentar la cantidad de ejercicio para conseguir el efecto deseado, o la disminución del efecto con el uso continuado de la misma cantidad), la abstinencia (manifestación de síntomas de abstinencia o necesidad de aumentar ejercicio para evitar dichos síntomas), la intención (se realiza más ejercicio del que se planea), la falta de control (deseo permanente e ineficaz de controlar o disminuir la cantidad de ejercicio realizado), el tiempo empleado (excesivo tiempo realizando ejercicio), la reducción de otras actividades (otras tareas como las ocupacionales o las recreacionales se ven afectadas por la realización de ejercicio) y la continuación (necesidad de seguir realizando ejercicio a pesar de problemas físicos, psicológicos o fisiológicos recurrentes provocados por el mismo ejercicio).

Esta escala ha mostrado ser fiable ( $\mathrm{r}=0.92$; Hausenblas y Downs, 2002) y válida, presentando aquellos sujetos con una mayor puntuación EDS-R más síntomas de dependencia al ejercicio (Downs, Hausenblas, y Nigg, 2004). Además, dicha escala presenta una alta consistencia interna para cada uno de los factores, con un valor $\alpha$ de 0,73 para la tolerancia; 0,85 para la abstinencia; 0,83 para la intención; 0,78 para la falta de control; 0,84 para el tiempo empleado; 0.68 para la reducción de otras actividades y 0,81 para la continuación, siendo el valor $\alpha$ para la consistencia global de la escala de 0,92 (Sicilia y González-Cutre, 2011). 
Valenzuela, P. L. y Arriba-Palomero, F. (2017). Riesgo de adicción al ejercicio en triatletas hombres amateur y su relación con variables de entrenamiento. RICYDE. Revista internacional de ciencias del deporte, 48(13), 162-171. https://doi.org/10.5232/ricyde2017.04806

Junto con este cuestionario se proporcionó a los participantes un anexo elaborado específicamente para este estudio en el que debían completar de forma anónima datos sobre variables de entrenamiento como los años de experiencia en el triatlón, su volumen de entrenamiento semanal medio en ese momento de la temporada (número de horas y sesiones por semana), y la distancia de mayor duración en la que normalmente competían (Sprint, Olímpica, Medio Ironman o Ironman).

\section{Procedimientos}

Durante un periodo no competitivo de la temporada de triatlón (Noviembre-Marzo) los investigadores acudieron a una sesión de entrenamiento y les proporcionaron personalmente, tras permiso de sus entrenadores, el cuestionario. Atendiendo a la EDS-R (Hausenblas y Downs, 2002), los sujetos fueron divididos en no dependientes asintomáticos (NDA), no dependientes sintomáticos (NDS), y en riesgo de dependencia (ERD). Es importante remarcar que, aunque esta escala indica el riesgo de un sujeto para desarrollar adicción al ejercicio, es tan solo una herramienta de cribado y por lo tanto son necesarios tests adicionales para realizar un diagnóstico final (Szabo y col, 2015). Los sujetos fueron también divididos en tres subgrupos atendiendo a la distancia en la que competían: Corta (aquellos que competían en distancia sprint y olímpica), Media (distancia medio Ironman) y Larga (distancia Ironman).

\section{Análisis estadístico}

Los datos se muestran como Media \pm SD. La normalidad y homocedasticidad de los datos fue comprobada de forma previa al análisis estadístico (Test de Levene). Se realizó un análisis de la varianza de una vía (ANOVA) con el posterior test Tukey HSD para determinar las diferencias entre grupos atendiendo al riesgo de dependencia (NDA, NDS y ERD) y a la distancia de competición (Corta, Media y Larga). Se estableció un valor mínimo de significancia de $p<0.05$ y máximo de $p<0.001$. Con el fin de examinar la magnitud de las diferencias encontradas entre grupos se calculó el tamaño del efecto ( $\mathrm{d}$ de Cohen), siendo un valor $d=0.2,0.5,0.8$ y 1.3 considerado pequeño, moderado, grande y muy grande, respectivamente. Además, se analizaron las correlaciones ( $r$ de Pearson) entre las distintas variables de entrenamiento y el riesgo de dependencia. El análisis estadístico fue realizado con el software Statgraphics Plus 5.1 (StatPoint, Inc., Virginina, USA).

\section{Resultados}

La puntuación media en la EDS-R fue de $57.18 \pm 17.31$. De los 93 triatletas analizados, un $8.6 \%$ fue clasificado como ERD $(81.6 \pm 5.9$ puntos $)$, un $60.2 \%$ como NDS $(64.3 \pm 13.4$ puntos) y un $31.2 \%$ como NDA (39.3 \pm 8.6 puntos).

\section{Variables de entrenamiento}

Se encontraron diferencias significativas con un gran tamaño del efecto entre los distintos grupos de dependencia tanto para el número de horas (Fig. 1A) como de sesiones semanales (Fig. 1B), estando ambas variables moderadamente correlacionadas con la puntuación EDS-R (Fig. 1C y 1D).

Hubo también diferencias significativas, aunque con un bajo tamaño del efecto $(p<0.05$, $d=0.3)$, en el número de años de experiencia de los grupos ERD $(8.63 \pm 5.07$ años $)$ y NDA ( $7.10 \pm 6.76$ años), pero no se encontraron diferencias significativas entre ERD y NDS (8.02 \pm 5.76 años) o entre NDS y NDA. Los años de experiencia no se correlacionaron de forma significativa con la puntuación EDS-R. 
Valenzuela, P. L. y Arriba-Palomero, F. (2017). Riesgo de adicción al ejercicio en triatletas hombres amateur y su relación con variables de entrenamiento. RICYDE. Revista internacional de ciencias del deporte, 48(13), 162-171. https://doi.org/10.5232/ricyde2017.04806

A

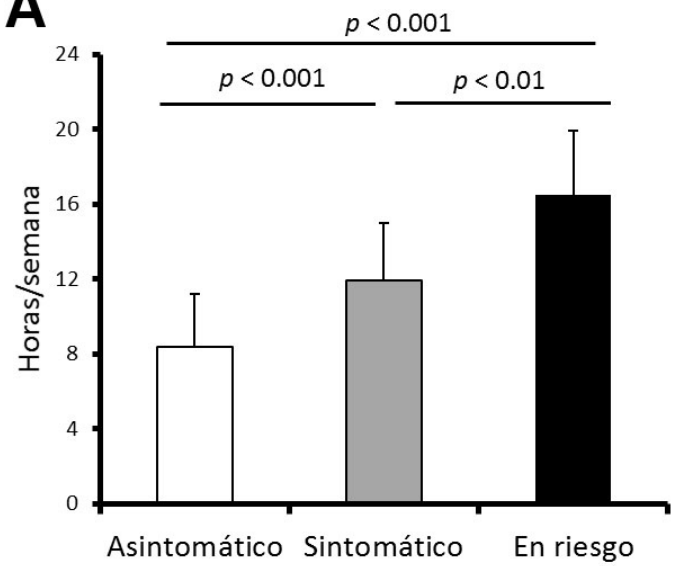

C

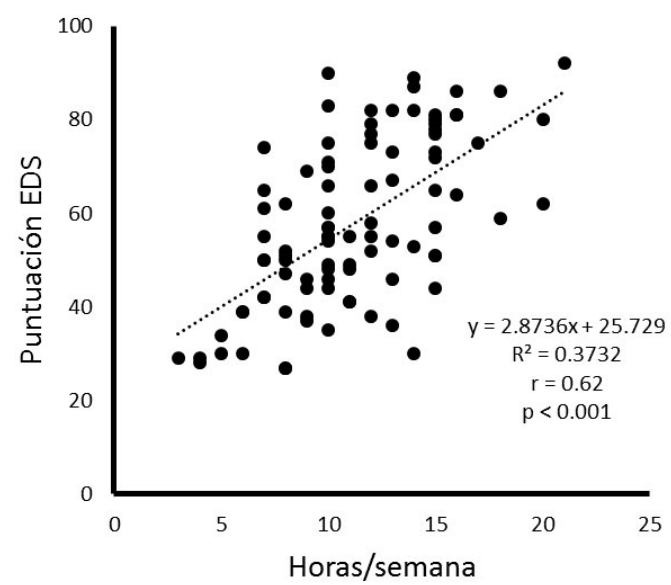

B
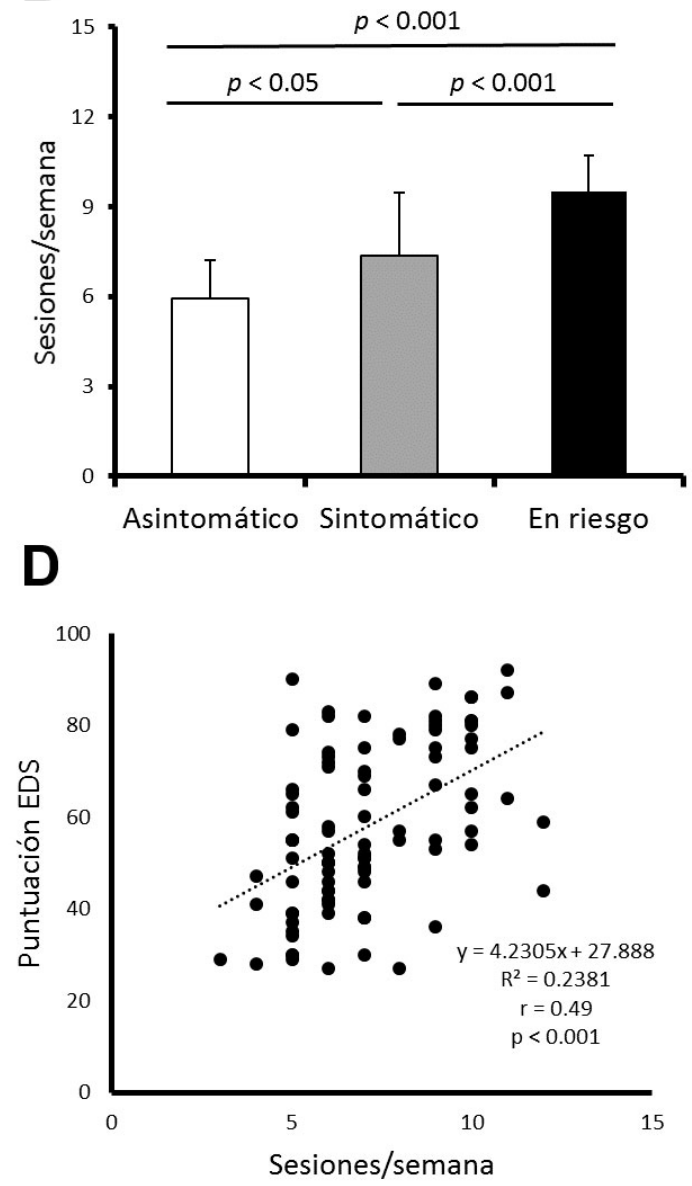

Figura 1: Número de horas de entrenamiento (A) y de sesiones a la semana (B) atendiendo al riesgo de adicción, y análisis de correlación entre ambas variables de entrenamiento y la puntuación en la escala EDS-R (C y D).

\section{Distancia de competición}

A

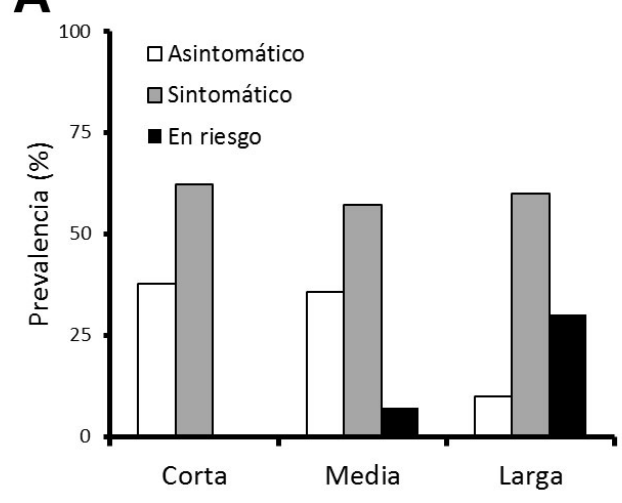

B

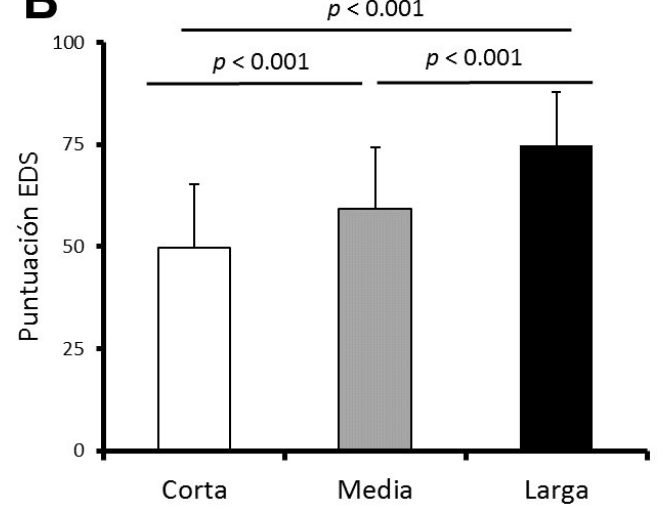

Figura 2: Porcentaje de sujetos en cada nivel de riesgo de adicción (A) y puntuación en la escala EDS-R (B) atendiendo a la distancia en la que competían los triatletas. 
Valenzuela, P. L. y Arriba-Palomero, F. (2017). Riesgo de adicción al ejercicio en triatletas hombres amateur y su relación con variables de entrenamiento. RICYDE. Revista internacional de ciencias del deporte, 48(13), 162-171. https://doi.org/10.5232/ricyde2017.04806

Se encontró una mayor prevalencia de riesgo de adicción (Fig. 2A) y una mayor puntuación EDS-R (Fig. 2B) según aumentaba la distancia de competición, estando esta variable altamente relacionada con la puntuación EDS-R $\left(p<0.001 ; r=0.55, R^{2}=29.98 \%\right)$.

Como puede observarse en la Tabla 1, los triatletas de larga distancia entrenaban un mayor número de horas y sesiones a la semana que los de media $(d=0.8$ y 1.9 para el número de horas y de sesiones, respectivamente) y corta distancia ( $d=1.6$ y 1.9 , respectivamente), siendo también significativas las diferencias para el número de horas y sesiones semanales entre estos últimos ( $d=0.9$ y 0.6 , respectivamente).

Se halló una correlación moderada entre la distancia de competición y el número de horas $\left(p<0.001 ; r=0.60 ; R^{2}=35.89 \%\right)$ y de sesiones de entrenamiento $\left(p<0.001, r=0.57, R^{2}=32.73 \%\right)$ por semana, así como una leve correlación entre la distancia de competición y los años de experiencia $\left(p<0.01, r=0.28, R^{2}=8.07 \%\right)$.

Tabla 1: Variables de entrenamiento atendiendo a la distancia en la que competían los triatletas

\begin{tabular}{|l|c|c|c|}
\hline & Corta & \multicolumn{1}{|c|}{ Media } & \multicolumn{1}{c|}{ Larga } \\
\hline Años de experiencia & $6.1 \pm 5.4$ & $8.8 \pm 6.3$ & $10.3 \pm 2.5$ \\
\hline Horas de entrenamiento/semana & $9.2 \pm 3.1$ & $11.9 \pm 2.9 * *$ & $14.9 \pm 4.0^{* * \#}$ \\
\hline Sesiones de entrenamiento/semana & $6.1 \pm 1.8$ & $7.2 \pm 1.9 *$ & $9.2 \pm 1.4 * * \#$ \\
\hline
\end{tabular}

Los datos se muestran como Media \pm SD. Diferencias significativas con respecto al grupo de corta distancia: ${ }^{*} \mathrm{p}<0.01,{ }^{*} \mathrm{p}<0.001$. Diferencias significativas con respecto al grupo de media distancia: ${ }^{\#} \mathrm{p}<0.01,{ }^{\#} \mathrm{p}<0.001$.

\section{Discusión}

Pese a que hasta el momento tan solo la adicción al juego está reconocida en el DSM como adicción conductual (Egorov y Szabo, 2013), otras actividades como el trabajo (Scottl, 1997), las compras (Black, 2007) y la televisión (Kubey y Csikszentmihalyi, 2002) han mostrado ser potencialmente adictivas, compartiendo los mismos mecanismos neurobiológicos que otras adicciones como la del alcohol o las drogas (Martin y Petry, 2005). El ejercicio físico, que normalmente es considerado como una actividad terapéutica a nivel físico y mental (Penedo y Dahn, 2005), es una de estos comportamientos que puede desembocar en adicción (Egorov y Szabo, 2013).

La prevalencia de adicción al ejercicio es muy variable entre los distintos estudios presentes en la literatura científica dependiendo de la población estudiada y el método de evaluación (Szabo y col. 2015). Por ejemplo, mientras que Mónok y col (2012) hallaron una prevalencia del $0.3-0.5 \%$ en la población general, este valor aumenta a $1.9-3.2 \%$ en la población deportivamente activa en el mismo estudio y hasta el $42 \%$ cuando otros autores analizaron a gente que entrenaba en gimnasio (Lejoyeux, Avril, Richoux, Embouazza, y Nivoli, 2008).

Algunos estudios previos han evaluado el riesgo de adicción al ejercicio en triatletas mostrando diferente prevalencia, desde el 8.4\% encontrado por Magee y col. (2016) al 20$30 \%$ encontrado por Blaydon y col. (2002) y Ruby (2008). Sin embargo, y aunque la población analizada fue en todos los casos un grupo de triatletas, éstos presentaban características diferentes. En el caso de Ruby (2008) y Magee y col. (2016) la muestra incluía triatletas de larga distancia, los cuales presentan un mayor riesgo de adicción como muestran otros autores (Youngman y Simpson, 2014) y como confirma el presente estudio. Cada distancia de competición en triatlón presenta unas características particulares (duración e intensidad) y unos requerimientos físicos específicos (Laursen, 2011). Por ello, no es 
Valenzuela, P. L. y Arriba-Palomero, F. (2017). Riesgo de adicción al ejercicio en triatletas hombres amateur y su relación con variables de entrenamiento. RICYDE. Revista internacional de ciencias del deporte, 48(13), 162-171. https://doi.org/10.5232/ricyde2017.04806

conveniente estudiar esta población de forma independiente a esta variable. Por otro lado, en el estudio de Blaydon y col. (2002) se analizaron tanto triatletas amateur como profesionales, presentando estos últimos puntuaciones mayores en los cuestionarios de adicción al ejercicio.

Youngman y Simpson (2014) determinaron la prevalencia de riesgo de adicción al ejercicio en triatletas atendiendo a la distancia en la que los sujetos competían, encontrando un 15, 28 y $23 \%$ de prevalencia de riesgo de adicción en los triatletas de corta, media y larga distancia respectivamente. En cuanto a las variables de entrenamiento, estos autores observaron que el riesgo de adicción se incrementaba desde distancia olímpica a media distancia, sugiriendo que este hecho podría ser debido al mayor volumen de entrenamiento de los últimos, lo que podría suponer conflictos interpersonales y conllevar una situación de irritabilidad o depresión si no podían entrenar. Sin embargo, dicho estudio no encontró diferencias entre triatletas de distancia olímpica y de larga distancia, por lo que no se halló una relación lineal entre riesgo de adicción y distancia de competición. Además, no recogieron datos sobre el volumen de entrenamiento de cada grupo de triatletas, por lo que su hipótesis no fue sustentada por resultados objetivos. En el presente estudio tanto el riesgo de adicción como el volumen de entrenamiento difirieron entre las distintas distancias de competición, estando un 7 y un 30\% de los triatletas de media y larga distancia en riesgo de adicción, pero sin triatletas de corta distancia en esta situación. Sin embargo, al contrario que Youngman y Simpson (2014), en el presente estudio se ha encontrado una relación lineal entre la distancia de competición, el volumen de entrenamiento, y el riesgo de adicción.

Una explicación para la relación entre el volumen de entrenamiento y el riesgo de adicción puede ser la hipótesis de la activación simpática (Sympathetic Arousal Hypothesis), formulada por Thompson y Blanton (1987). Según esta teoría, cuando los sujetos se acostumbran a realizar una cierta cantidad de ejercicio la actividad simpática del organismo se reduce, experimentando así un estado letárgico. Por ello, los triatletas incrementarían su volumen de ejercicio para incrementar el estado de activación, pero al ser este efecto temporal necesitarían incrementar además la frecuencia de entrenamiento para cumplir su objetivo. No obstante, es importante remarcar que altos volúmenes de entrenamiento no son necesariamente problemáticos, sino que pueden ser tan solo un resultado de las demandas físicas del deporte y no un patrón patológico de ejercicio (Cook, Karr, Zunker, Mitchell, Thompson, Sherman, Crosby, Cao, Erickson, y Wonderlich, 2013).

Por otro lado, la relación encontrada entre la distancia de competición y el volumen de entrenamiento, aunque puede deberse en parte a una mayor necesidad de realizar ejercicio según aumenta la distancia por la mayor prevalencia de adicción al ejercicio, está asociada seguramente en gran medida a la necesidad de los deportistas de asimilar su entrenamiento a las condiciones en las que competirán, aumentando así el volumen de entrenamiento según aumenta la distancia de competición de acuerdo al principio de especificidad.

Por lo tanto, se puede concluir que competir en larga distancia y aumentar el volumen de entrenamiento -lo cual puede ser una consecuencia de lo anterior- son factores de riesgo a tener en cuenta, aunque no se puede discernir si el mayor volumen de entrenamiento presentado por los triatletas en riesgo de adicción es una causa o una consecuencia de esta patología.

Es importante remarcar que, pese a que algunos estudios han analizado el riesgo de adicción en diferentes poblaciones, pocos han estudiado la adicción en sí, para lo cual es necesario realizar entrevistas personales tras el cribado inicial como muestran Müller, Cook, Zander, Herberg, Müller y Zwaan (2014). No obstante, la prevalencia de riesgo de adicción encontrada en el presente estudio y en otros realizados en triatletas (Blaydon y col. 2002; 
Valenzuela, P. L. y Arriba-Palomero, F. (2017). Riesgo de adicción al ejercicio en triatletas hombres amateur y su relación con variables de entrenamiento. RICYDE. Revista internacional de ciencias del deporte, 48(13), 162-171. https://doi.org/10.5232/ricyde2017.04806

Magee y col. 2016; Ruby, 2008; Youngman y Simpson, 2014) muestra valores superiores a los encontrados en otras poblaciones (Allegre, Therme, y Griffiths, 2007), siendo además el porcentaje de triatletas no dependientes pero sintomáticos también preocupante $(60.2 \%)$. Por ello, consideramos que estos resultados deben ser tenidos en cuenta para crear consciencia del potencial adictivo del triatlón entre los entrenadores o psicólogos del ejercicio, de forma que presten especial atención a sus deportistas y más aún ante un aumento del volumen de entrenamiento o si compiten en larga distancia.

\section{Conclusiones}

El presente estudio muestra una importante prevalencia de riesgo de adicción en triatletas amateur (8.6\%), siendo este valor mayor que el de otras poblaciones analizadas en la literatura científica. Además, atendiendo a estos resultados, el riesgo de adicción es dependiente del volumen de entrenamiento y de la distancia en la que los triatletas compiten, alcanzando una prevalencia del $30.0 \%$ en el caso de los triatletas de larga distancia.

Consideramos que el tamaño muestral utilizado puede ser una limitación del estudio, aunque este hecho ha sido provocado en parte por la búsqueda de homogeneidad en el grupo. No obstante, consideramos que éste factor debería ser mejorado en futuros estudios, los cuales podrían analizar también la prevalencia de riesgo de adicción al ejercicio atendiendo a otras variables como el sexo, el carácter profesional o amateur del triatleta, el momento de la temporada o la pertenencia o no a un grupo de entrenamiento guiado por especialistas.

A pesar de las limitaciones nombradas, estos resultados ponen de manifiesto el importante papel del psicólogo del ejercicio y el entrenador para la prevención de la adicción al ejercicio en el deportista. Los profesionales del ejercicio deben ser conscientes del potencial adictivo del triatlón, especialmente de larga distancia, y de la influencia que tiene el volumen de entrenamiento en el desarrollo de dicha adicción, pudiendo establecer así protocolos para su prevención o detección temprana (cuestionarios como el utilizado en este estudio, conversación con el deportista, observar síntomas, etc.).

\section{Agradecimientos}

Queremos mostrar nuestro agradecimiento a los entrenadores por su apoyo técnico así como a los triatletas por su participación en este estudio. Este trabajo no ha recibido financiación.

\section{Referencias}

Allegre, B.; Therme, P., \& Griffiths, M. (2007). Individual factors and the context of physical activity in exercise dependence: A prospective study of "ultra-marathoners." International Journal of Mental Health and Addiction, 5(3), 233-243. https://doi.org/10.1007/s11469-007-9081-9

Berczik, K.; Szabó, A.; Griffiths, M. D.; Kurimay, T.; Kun, B.; Urbán, R., \& Demetrovics, Z. (2012). Exercise Addiction: Symptoms, Diagnosis, Epidemiology, and Etiology. Substance Use \& Misuse, 47, 403-417. https://doi.org/10.3109/10826084.2011.639120

Black, D. W. (2007). Compulsive buying disorder: a review of the evidence. World Psyquiatry, 6(1), 14-18.

https://doi.org/10.1017/s1092852900020630 
Valenzuela, P. L. y Arriba-Palomero, F. (2017). Riesgo de adicción al ejercicio en triatletas hombres amateur y su relación con variables de entrenamiento. RICYDE. Revista internacional de ciencias del deporte, 48(13), 162-171. https://doi.org/10.5232/ricyde2017.04806

Blaydon, M. J.; Lindner, K. J., \& Kerr, J. H. (2002). Metamotivational characteristics of eating-disordered and exercise-dependent triathletes: An application of reversal theory. Psychology of Sport and Exercise, 3, 223-236.

https://doi.org/10.1016/S1469-0292(01)00021-8

Cook, B.; Karr, T. M.; Zunker, C.; Mitchell, J. E.; Thompson, R.; Sherman, R.; Crosby, R. D.; Cao, L.; Erickson, A., \& Wonderlich, S. A. (2013). Primary and secondary exercise dependence in a community-based sample of road race runners. Journal of sport \& exercise psychology, 35(5), 464-469.

https://doi.org/10.1123/jsep.35.5.464

Downs, D. S.; Hausenblas, H. A., \& Nigg, C. R. (2004). Factorial validity and psychometric examination of the exercise dependence scale-revised. Measurement in physical education and exercise science, 8(4), 183-201.

https://doi.org/10.1207/s15327841mpee0804_1

Egorov, A. Y., \& Szabo, A. (2013). The exercise paradox: An interactional model for a clearer conceptualization of exercise addiction. Journal of Behavioral Addictions, 2(4), 199-208. https://doi.org/10.1556/JBA.2.2013.4.2

Fiuza-Luces, C.; Garatachea, N.; Berger, N. A., \& Lucia, A. (2013). Exercise is the real polypill. Physiology, 28, 330-58.

https://doi.org/10.1152/physiol.00019.2013

Griffiths, M. (1997). Exercise addiction. A case study. Addiction Research, 5(2), 161-168. https://doi.org/10.3109/16066359709005257

Hausenblas, H. A., \& Downs, D. S. (2002). How much is too much? The development and validation of the exercise dependence scale. Psychology \& Health, 17(4), 387-404. https://doi.org/10.1080/0887044022000004894

Heazlewood, I., \& Burke, S. (2011). Self-efficacy and its relationship to selected sport psychological constructs in the prediction of performance in ironman triathlon. Journal of Human Sport and Exercise, 6(2), 328-350.

https://doi.org/10.4100/jhse.2011.62.14

Kubey, R., \& Csikszentmihalyi, M. (2002). Television addiction is not mere metaphore. Scientific American, 286(2), 74-81.

https://doi.org/10.1038/scientificamerican0202-74

Laursen, P. B. (2011). Long distance triathlon: demands, preparation and performance. Journal of Human Sport and Exercise, 6(2), 247-263.

https://doi.org/10.4100/jhse.2011.62.05

Lejoyeux, M.; Avril, M.; Richoux, C.; Embouazza, H., \& Nivoli, F. (2008). Prevalence of exercise dependence and other behavioral addictions among clients of a Parisian fitness room. Comprehensive Psychiatry, 49(4), 353-358.

https://doi.org/10.1016/j.comppsych.2007.12.005

Magee, C. A.; Buchanan, I., \& Barrie, L. (2016). Profiles of exercise dependence symptoms in Ironman participants. Psychology of Sport and Exercise, 24, 48-55. https://doi.org/10.1016/j.psychsport.2016.01.005

Martin, P. R., \& Petry, N. M. (2005). Are non-substance-related addictions really addictions? The American journal on addictions / American Academy of Psychiatrists in Alcoholism and Addictions, 14(1), 1-7.

McNamara, J., \& McCabe, M. P. (2012). Striving for success or addiction? Exercise dependence among elite Australian athletes. Journal of Sports Sciences, 30(8), 755766.

https://doi.org/10.1080/02640414.2012.667879 
Valenzuela, P. L. y Arriba-Palomero, F. (2017). Riesgo de adicción al ejercicio en triatletas hombres amateur y su relación con variables de entrenamiento. RICYDE. Revista internacional de ciencias del deporte, 48(13), 162-171. https://doi.org/10.5232/ricyde2017.04806

Mónok, K.; Berczik, K.; Urbán, R.; Szabo, A.; Griffiths, M. D.; Farkas, J.; Magi, A.; Eisinger, A.; Kurimay, T.; Kökönyei, G.; Kun, B.; Paksi, B., \& Demetrovics, Z. (2012). Psychometric properties and concurrent validity of two exercise addiction measures: $A$ population wide study. Psychology of Sport and Exercise, 13, 739-746. https://doi.org/10.1016/j.psychsport.2012.06.003

Müller, A.; Cook, B.; Zander, H.; Herberg, A.; Müller, V., \& de Zwaan, M. (2014). Does the German version of the Exercise Dependence Scale measure exercise dependence? Psychology of Sport and Exercise, 15(3), 288-292. https://doi.org/10.1016/j.psychsport.2012.06.003

Penedo, F. J., \& Dahn, J. R. (2005). Exercise and well-being: a review of mental and physical health benefits associated with physical activity. Current opinion in psychiatry, 18, 189-193.

https://doi.org/10.1097/00001504-200503000-00013

Ruby, A. L. (2008). In sickness and in health: Exercise addiction and the Ironman triathlete. The university of Iowa.

Scottl, K. S. (1997). An Exploration of the Meaning and Consequences of Workaholism. Human Relations, 50, 287-314. https://doi.org/10.1177/001872679705000304

Sicilia, A., \& González-Cutre, D. (2011). Dependence and physical exercise: Spanish validation of the Exercise Dependence Scale-Revised (EDS-R). The Spanish Journal of Psychology, 4(1), 421-431.

https://doi.org/10.5209/rev_SJOP.2011.v14.n1.38

Slay, H. A.; Hayaki, J.; Napolitano, M. A., \& Brownell, K. D. (1998). Motivations for running and eating attitudes in obligatory versus nonobligatory runners. International Journal of Eating Disorders, 23(3), 267-275. https://doi.org/10.1002/(SICI)1098-108X(199804)23:3<267::AID-EAT4>3.0.CO;2-H

Szabo, A.; Griffiths, M. D.; de La Vega Marcos, R.; Mervó, B., \& Demetrovics, Z. (2015). Methodological and conceptual limitations in exercise addiction research. Yale Journal of Biology and Medicine, 88(3), 303-308.

Thompson, J. K., \& Blanton, P. (1987). Hypothesis, Energy conservation and exercise dependence: a sympathetic arousal. Medicine \& Science in Sports \& Exercise, 19(2), 91-99. https://doi.org/10.1249/00005768-198704000-00005

Vleck, V.; Millet, G. P., \& Alves, F. B. (2014). The impact of triathlon training and racing on athletes' general health. Sports Medicine, 44(12), 1659-1692. https://doi.org/10.1007/s40279-014-0244-0

Youngman, J., \& Simpson, D. (2014). Risk for exercise addiction: A comparison of triathletes training for sprint-, olympic-, half-ironman-, and ironman-distance triathlons. Journal of Clinical Sport Psychology, 8, 19-37.

https://doi.org/10.1007/s40279-014-0244-0 